\title{
A Patient with a Prolonged Activated Partial Thromboplastin Time and a Deep Intracerebral Haemorrhage
}

\author{
Roger K. Schindhelm ${ }^{a, b} \quad$ Mariëlle J. Wondergem ${ }^{f}$ \\ Joke Admiraal ${ }^{c}$ Gert Nap $^{d}$ Edwin ten Boekel ${ }^{a, b}$ \\ Lahcen $\mathrm{Hani}^{\mathrm{e}}$ \\ ${ }^{a}$ Department of Clinical Chemistry, Haematology and Immunology, Medical \\ Centre Alkmaar, Alkmaar, Departments of ${ }^{b}$ Clinical Chemistry and \\ Haematology, 'Internal Medicine, ${ }^{\mathrm{d}}$ Hospital Pharmacy and ${ }^{\mathrm{e}}$ Neurology, Gemini \\ Hospital, Den Helder, and ' Department of Haematology, VU University Medical \\ Centre, Amsterdam, The Netherlands
}

\section{Key Words}

Intracerebral haemorrhage $\cdot$ Partial thromboplastin time $\cdot$ Mixing studies Factor XII deficiency

\begin{abstract}
We report on a 57-year-old woman with a pontine haemorrhage and an extremely prolonged activated partial thromboplastin time (aPTT) of more than $240 \mathrm{~s}$, suggestive of a coagulation disorder. Given the location of the haemorrhage, which is associated with a high mortality rate, recombinant factor VIla was administered, although not all necessary laboratory analyses could be performed at that time. In our case, a deficiency of factor XII was found, which is not associated with an increased bleeding risk. In an acute setting, evaluation of a prolonged aPTT may cause diagnostic and therapeutic challenges, in particular in situations where additional laboratory investigations may not be readily available.
\end{abstract}

\section{Introduction}

Prothrombin time (PT) and activated partial thromboplastin time (aPTT) are the most common ordered coagulation tests for the screening of coagulation disorders. PT 
and aPTT are prolonged in case of a coagulation factor deficiency or an acquired inhibitor (i.e. antibody against a clotting factor) [1]. In patients presenting with stroke, both tests are ordered in the initial evaluation of coagulation disorders. Indeed, coagulation disorders may be related to an increased risk of intracerebral haemorrhage (ICH) by either initiating or contributing to bleeding [2]. We report a patient with an intracerebral pontine bleeding and a prolonged aPTT. In this patient, the cause of the prolonged aPTT and the relation to ICH were not clear during the acute phase of the disease.

\section{Case Report}

A 57-year-old woman presented to the emergency department $1.5 \mathrm{~h}$ after the acute onset of paresthaesia of the right arm and tingling of the mouth. She had a history of recently diagnosed hypertension and migraine. At presentation, her blood pressure was 193/88 $\mathrm{mm} \mathrm{Hg}$ and the initial symptoms were already partially abated. The neurologic evaluation revealed only an atactic gait and diminished pin prick sensation of the right fore arm. The ECG showed no abnormalities. Laboratory results were as follows: PT = 13 s (reference values: 9-14), aPTT >240 s (reference values: 24-35). Platelet count and haemoglobin content were within the reference range. To rule out possible preanalytical or analytical errors as a cause of the prolonged aPTT, a new blood sample was drawn. However, similar results were obtained (aPTT $=226$ and $>240$ s). To our knowledge, no heparin was administered to the patient. CT and MRI scans taken within $3 \mathrm{~h}$ of the onset of the symptoms showed a small pontine bleeding (fig. 1). Because of the location of ICH (pontine bleeding), which is associated with a high mortality rate $[2,3]$ combined with the prolonged aPTT probably indicating a coagulation disorder, urgent action had to be taken. The patient had no known (family) history of bleeding and haemostasis had never been tested before. Since relevant additional laboratory investigations could not be performed on a stat basis in the hospital's laboratory, the consulting haematologist advised administration of one dose of recombinant factor VIIa (NovoSeven ${ }^{\circledR}, 90 \mu \mathrm{g} / \mathrm{kg}$ ), even though not all necessary analyses were performed at that time. This was done to treat a possible acquired factor VIII or factor IX inhibitor. This might cause an extremely prolonged aPTT, but often goes combined with spontaneous bleeding or haematomas and progression of ICH. The patient was admitted to the stroke unit for administration of recombinant factor VIIa and further observation. The same night, $6 \mathrm{~h}$ after presentation, the necessary additional tests were performed in an external coagulation laboratory. To exclude the presence of heparin in the blood sample, a thrombin time was performed by the external coagulation laboratory. Thrombin time was not prolonged, indicating that heparin administration or contamination could be excluded as a cause of the prolonged aPTT. The aPTT mixing test was suggestive for a factor deficiency as a cause of the prolonged aPTT ( $\underline{\text { table } 1}$ and fig. 2). Activity of factor VIII and factor IX was determined in a sample drawn before recombinant factor VIIa was administered. Both results were within the reference range. An irrelevant factor deficiency was suspected and confirmed the following day when further laboratory testing showed an extremely low factor XII activity ( $<6 \%$, reference value $>60 \%$ ). During the following days, our patient's neurological condition improved and she showed almost complete recovery. She was discharged after 4 days. The patient has given informed consent for the publication of this case report.

\section{Discussion}

In patients presenting with stroke, only a minority (10-15\%) have stroke caused by ICH. Primary ICH makes up approximately $70-80 \%$ of all ICH cases. Chronic hypertension is the most common cause of ICH. It commonly affects the cerebral lobes, basal ganglia, thalamus, brain stem (predominantly the pons), and the cerebellum as a result of ruptured vessels. The exact frequency of a pontine bleeding as the primary location of ICH is not known, but it is estimated to be less than $10 \%$ [3]. Depending on 
the extent of the haematoma, a pontine bleeding can result in a coma and total paralysis and is associated with a high mortality rate (up to $85 \%$ ) within the first $48 \mathrm{~h}$ [2]. Therefore, optimal diagnostic and treatment modalities should be initiated. Evaluation of a bleeding disorder should be performed in every patient with ICH, especially if the cause is not immediately obvious. Although hypertension is the most prevalent cause of ICH, accounting for $70-80 \%$ of all cases, a bleeding disorder may initiate or contribute to bleeding resulting in a high mortality rate. During acute presentation it is not always possible to perform additional laboratory tests to evaluate the cause of the prolonged aPTT. An aPTT mixing study should be performed as soon as possible to differentiate between a coagulation factor deficiency and an inhibitor (fig. 2) since treatment differs markedly. aPTT is an indicator measuring the efficacy of the contact activation pathway and the common coagulation pathway, and includes coagulation factors XII, XI, IX, VIII, X, V, prothrombin, and fibrinogen (fig. 2). Contact activation, which only occurs in vitro, starts with factor XII in conjunction with prekallikrein and high molecular weight kininogen. In vivo, the coagulation cascade is initiated by tissue factor and factor VII, and activation via factor XII is of no relevance. In case of a prolonged aPTT artefact such as lipeamic or icteric plasma, high haematocrit $(>0.60 \mathrm{l} / \mathrm{l})$ or a delay between venipuncture and aPTT measurement should be excluded. Furthermore, heparin contamination of the test tube can cause a false prolonged aPTT. In case of doubt, a new blood sample obtained by venipuncture should be taken and analyzed as soon as possible. In the evaluation of a prolonged aPTT in case the aPTT mixing study (fig. 2) is indicative of a factor deficiency, a deficiency in one or more of the aforementioned (coagulation) factors should be considered (table 1). Of note, a low level of von Willebrand factor, the carrier protein of factor VIII, is also reflected in a prolonged aPTT. A factor XII deficiency results in a markedly prolonged aPTT in vitro, but is not associated with a bleeding tendency in vivo [1]. The prevalence of severe factor XII deficiency (factor XII activity $<20 \%$ ) in the general population is estimated to be less than $0.5 \%$, whereas severe to moderate factor XII activity (20-45\%) is found in $1-3 \%$ of all individuals [4]. A factor XI deficiency is very rare (prevalence: 1 per million) and is associated with a symptomatic bleeding tendency depending on the genetic mutation $[5,6]$. In case the aPTT mixing study is indicative of a coagulation factor inhibitor, an inhibitor of factor VIII or factor IX or a lupus anticoagulant should be considered. Lupus anticoagulant is associated with an increased risk of thrombosis. The incidence of an acquired factor VIII inhibitor is very low and estimated to be 1-4 per million per year [7]. Acquired factor IX inhibitors are even more infrequent, and thus data on their incidence are unavailable [8]. Although these autoantibodies are very uncommon, morbidity and mortality are relatively high. Symptomatic bleeding may occur in up to $90 \%$ of these patients with a mortality rate up to $22 \%$ [7]. A factor VIII or factor IX inhibitor causes a moderate to markedly prolonged aPTT and testing should be aimed at the assay of the activity of these coagulation factors and the inhibitor activity. In most nonacademic hospital laboratories (including ours), the assays of the separate coagulation factors including factors VIII, IX, XI, and XII cannot be performed on a stat basis. Moreover, since factor XII deficiency has no clinical consequences, laboratories do not have procedures allowing stat factor XII testing. Management in the acute setting of ICH is aimed at reducing the expansion of the haematoma. Recombinant factor VIIa (NovoSeven) has been used in patients with ICH. A recent meta-analysis suggests that treatment with recombinant factor VIIa may limit the growth of the haematoma, but no improvement in clinical outcome has been seen [9]. However, most 
series do not report on patients with a known prolonged aPTT in whom bleeding risk might be higher. Moreover, one should realize that the use of recombinant factor VIIa is associated with an increase in arterial and venous thromboembolic events, and thus unnecessary use should be limited. Fresh frozen plasma (FFP) is indicated in patients with coagulation factor deficiencies. In case of coagulation factor inhibitors, FFP is an inadequate haemostatic therapy since the factor levels are insufficient to overcome the antibody titre of the inhibitor. Therefore, FPP is not the optimal line of therapy in patients with autoantibodies against factor VIII or factor IX, and thus bypassing agents such as NovoSeven are preferred [10].

In conclusion, we reported on a patient with ICH and a markedly prolonged aPTT in whom a deficiency of factor XII was found, which was not associated with an increased bleeding tendency. In an acute setting, this may cause diagnostic and therapeutic challenges, in particular in situations where additional laboratory investigations may not be readily available.

\section{Disclosure Statement}

The authors have no conflict of interest.

$\underline{\text { Table 1. }}$. Causes of a prolonged aPTT with a PT within the reference range

\begin{tabular}{ll}
\hline With bleeding disorder & Without bleeding disorder \\
\hline Factor VIII (Haemophilia A) & Lupus anticoagulant \\
Factor IX (Haemophilia B) & Factor XII deficiency \\
Factor XI (Haemophilia C) & Factor XI deficiency (Haemophilia C) \\
Heparin therapy & PK deficiency \\
& HMWK deficiency \\
& von Willebrand factor deficiency \\
& Artefact
\end{tabular}

HMWK = High molecular weight kininogen; PK = prekallikrein. 

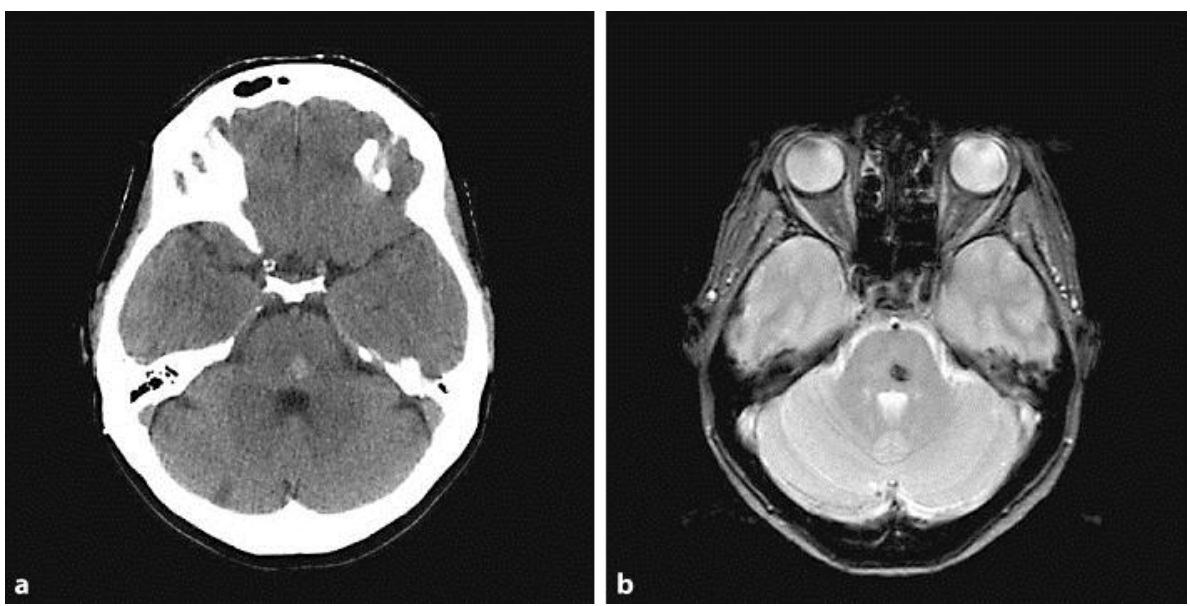

Fig. 1. A CT scan (a) and a gradient echo sequence MRI scan (b) with a small left paramedian pontine haemorrhage.

$\underline{\text { aPTT }}$

CONTACT FACTORS, FXI, FXII

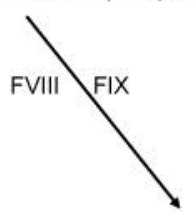

FX, FV, PHOSPHOLIPIDS

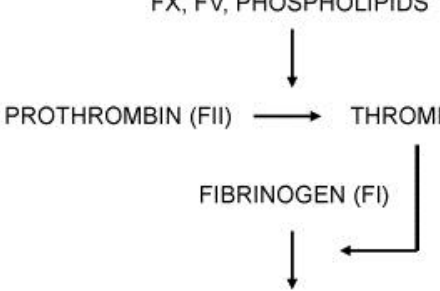

INITIAL FIBRIN CLOT
$\underline{\mathrm{PT}}$

TISSUE FACTOR

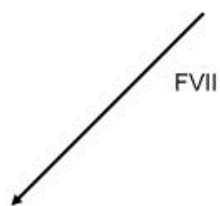

FVII

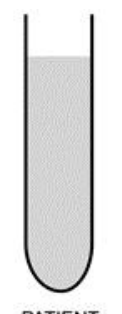

PATIENT

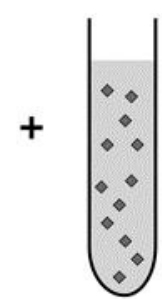

NORMAL

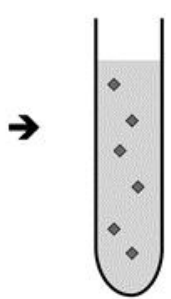

CORRECTED

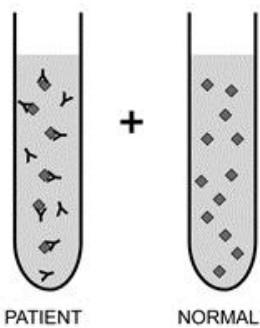

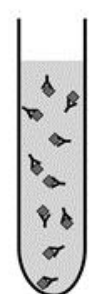

NOT CORRECTED

Fig. 2. Coagulation mechanisms as measured by PT and aPTT (left panel) and the principle of the mixing studies (right panel). In case of a factor deficiency, mixing of the patient plasma with normal pool plasma will result in the correction of the prolonged aPTT since a coagulation factor activity of $50 \%$ yields a normal (nonprolonged) aPTT (upper right panel). In case of a coagulation factor inhibitor, mixing of the patient plasma with normal plasma will not result in the correction of the aPTT since the inhibiting antibodies will also inhibit the coagulation factors in the plasma of the normal plasma (lower right panel). 


\section{References}

$\checkmark 1$ Kamal AH, Tefferi A, Pruthi RK: How to interpret and pursue an abnormal prothrombin time, activated partial thromboplastin time, and bleeding time in adults. Mayo Clin Proc 2007;82:864-873.

2 del Zoppo GJ, Mori E: Hematologic causes of intracerebral hemorrhage and their treatment. Neurosurg Clin N Am 1992;3:637-658.

-3 Thrift AG, Donnan GA, McNeil JJ: Epidemiology of intracerebral hemorrhage. Epidemiol Rev 1995;17:361-381.

-4 Lämmle B, Wuillemin WA, Huber I, Krauskopf M, Zürcher C, Pflugshaupt R, Furlan M: Thromboembolism and bleeding tendency in congenital factor XII deficiency - a study on 74 subjects from 14 Swiss families. Thromb Haemost 1991;65:117-121.

5 Gomez K, Bolton-Maggs P: Factor XI deficiency. Haemophilia 2008;14:1183-1189.

-6 Santoro R, Prejanò S, Iannaccaro P: Factor XI deficiency: a description of 34 cases and literature review. Blood Coagul Fibrinolysis 2011;22:431-435.

7 Franchini M, Lippi G: Acquired factor VIII inhibitors. Blood 2008;112:250-255.

8 Krishnamurthy P, Hawche C, Evans G, Winter M: A rare case of an acquired inhibitor to factor IX. Haemophilia 2011;17:712-713.

9 Yuan ZH, Jiang JK, Huang WD, Pan J, Zhu JY, Wang JZ: A meta-analysis of the efficacy and safety of recombinant activated factor VII for patients with acute intracerebral hemorrhage without hemophilia. J Clin Neurosci 2010;17:685-693.

10 Bouvry P, Recloux P: Acquired hemophilia. Haematologica 1994;79:550-556. 